(C) Blackwell Publishers Ltd. 1999, 108 Cowley Road, Oxford OX4 1JF, UK and 350 Main Street, Malden, MA 02148, USA.

Ratio (new series) XII 3 September $19990034-0006$

\title{
HOW MATHEMATICS ISN'T LOGIC
}

\author{
Roger Wertheimer
}

\begin{abstract}
If logical truth is necessitated by sheer syntax, mathematics is categorially unlike logic even if all mathematics derives from definitions and logical principles. This contrast gets obscured by the plausibility of the Synonym Substitution Principle implicit in conceptions of analyticity: synonym substitution cannot alter sentence sense. The Principle obviously fails with intercepting: nonuniform term substitution in logical sentences. 'Televisions are televisions' and 'TVs are televisions' neither sound alike nor are used interchangeably. Interception synonymy gets assumed because logical sentences and their synomic interceptions have identical factual content, which seems to exhaust semantic content. However, intercepting alters syntax by eliminating term recurrence, the sole strictly syntactic means of ensuring necessary term coextension, and thereby syntactically securing necessary truth. Interceptional necessity is lexical, a notational artifact. The denial of interception nonsynonymy and the disregard of term recurrence in logic link with many misconceptions about propositions, logical form, conventions, and metalanguages. Mathematics is distinct from logic: its truth is not syntactic; it is transmitted by synonym substitution; term recurrence has no essential role. The '=' of mathematics is an objectual relation between numbers; the '=' of logic marks a syntactic relation of coreferring terms.
\end{abstract}

Suppose that mathematics is analytic, that all arithmetic and other mathematical truths are derivable with only a handful of definitions and logical principles. Imagine a fallacy in Gödel's famous proof is found. Still, if logic is strictly formal, truth due solely to syntax, the real, radical difference between logic and mathematics remains. ${ }^{1}$

1 The thesis is conditional. I assume the classical conception of logic has been of truth internal to the form of thought (now called syntax), independent of its matter (meaning and reference of terms.) I don't justify that assumption or the conception. The thesis is confined to that conception's consequences, which may, incidentally, help justify the conception. The stated contrast of logic ('the science of thought, of truth') with mathematics, specifically arithmetic ('the science of number/quantity') extends to other $a$ priori disciplines: geometry, set theory, mereology. Also, the conception concerns logical truths (and falsehoods); semantically empty symbols of formal systems are another matter.

(C) Blackwell Publishers Ltd. 1999 
The notion of logico-syntactic form has obscurities aplenty that we needn't pause for; we can work with a few paradigms:

$$
(\mathrm{x})(\mathrm{Fx} \equiv \mathrm{Fx}) \quad \mathrm{Fav} \sim \mathrm{Fa} \quad \mathrm{Fa} \rightarrow \mathrm{Fa} \quad \sim(\mathrm{Fa \&} \sim \mathrm{Fa}) \quad \mathrm{a}=\mathrm{a} .
$$

Sentences having some such structure are logical because their syntax necessitates their truth, whatever their ' $F$ ' and ' $a$ ' terms mean or refer to. Sentences like:

$$
(\mathrm{x})(\mathrm{Fx} \equiv \mathrm{Gx}) \quad \mathrm{Fav} \sim \mathrm{Ga} \quad \mathrm{Fa} \rightarrow \mathrm{Ga} \quad(\mathrm{Fa} \& \sim \mathrm{Ga}) \quad \mathrm{a}=\mathrm{b}
$$

are extralogical whatever their terms mean, because their truth depends on what their predicates and names mean.

Syntactic form can survive uniform term substitution in any sentence, and nonuniform term substitution in extralogical sentences, but not intercepting: nonuniform substituting in a recurrence of a term pivotal in the truth-securing structure of a logical sentence. ${ }^{2}$ ' $37=$ XXXVII' is extralogical, not form ' $\mathrm{a}=\mathrm{a}$ ', not true by syntax.

All this should be beyond question, yet it has long been widely denied, sometimes explicitly, more often implicitly, most obviously, but not exclusively, in conceptions of analyticity going back through Frege and Kant to Locke. The denial is demanded by the seemingly platitudinous Synonym Substitution Principle. synonym substitution saves sentence sense. The Principle works the same whether synonymy is simply coreference or some other semantic equivalence. ${ }^{3}$

The Principle sounds platitudinous enough to be a definition of synonymy, until its exceptions are exposed. Synonym substitution in quotations, in intensional contexts like belief reports, ${ }^{4}$ and in self-referential sentences ${ }^{5}$ can alter sentence meaning and truth value. Intercepting is another exception, and of greater import. ${ }^{6}$ Yet the nonsynonymy of logical sentences with their interceptions went unrecognized until hardly half a century ago, and remains unpopular. ${ }^{7}$ That's astounding considering the plain

\footnotetext{
' 2 The inference in ' $\mathrm{p} \rightarrow(\mathrm{pvq})$ ' and '(p\&q) $\rightarrow \mathrm{p}$ ' pivots around 'p'. Whatever be sentence ' $\mathrm{q}$ ', subtracting it from the premises and adding it to the conclusion does not enter or alter the explanation of the necessity of the tautological conditional.

See my 'Identity: Logic, Ontology, Epistemology', Philosophy 73 (1998), pp. 179-93.

4 Benson Mates, 'Synonymity', University of California Publications in Philosophy 25 (1950), pp. 201-206.

William D. Hart, 'Self-Reference', Philosophical Review 79 (1970), pp. 523-8.

6 See my 'The Synonymy Antinomy', Proceedings of the Twentieth World Congress of Philosophy, Vol VI: Analytic Philosophy and Logic (Bowling Green, OH: Philosophy Documentation Center, 1999), and my 'Identity Syntax', op. cit., Vol. II: Metaphysics.

7 The first recorded heresies are Max Black's 'The "Paradox of Analysis" ' (Mind 53 (1944)) and Hilary Putnam's 'Synonymity and the Analysis of Belief Sentences' (Analysis $14(1954))$.
} 
fact that interception nonsynonymy blares in the ear of any competent speaker with a linguistic sense uncorrupted by semantic theory. Just listen:

VPL: A vice-president is a vice-president

VPI: VP is a vice-president

BBL: Either it's Batman or it's not Batman

BBI: Either it's Batman or it's not Bruce.

You don't need (and likely don't have) any idea what the sense difference is to be righteously certain such sentence pairs don't sound synonymous. The source of that certainty is not some suspected nonsynonymy of terms. For us participants in the public understanding that 'VP' abbreviates 'vice-president' there can be no sensible doubt of their (cognitive, informational) synonymy.

The sentences sound unlike because of the more basic, but no less blatant contrast in their usage. We don't standardly use VPL to say what we use VPI to say. 'A vice-president is a vice-president' does not say that the expression, 'VP', means vice-president, that 'VP' means what 'vice-president' means. Yet that is precisely what utterances of VPI are standardly used to say. Further, neither VPL nor VPI is normally used to say that 'vice-president' means vice-president.

Faith in interception synonymy happily flies in the face of all evidence, but is rarely openly avowed or verbalized at all. Commitment gets expressed in serene acceptance of its corollaries, for example, in untroubled talk of synomic interceptions being 'logically necessary', and their negations being '(self-)contradictions', despite lacking forms like 'p and $\sim \mathrm{p}$ ', ' $\sim(\mathrm{p}$ or $\sim \mathrm{p})$ ' or ' $\sim(\mathrm{a}=\mathrm{a})$ '. Worries about some of this sometimes surface. Some theorists balk at talk of interceptions being synonyms of their logical truths, but they go on calling them logical truths. In the dialect of other dialecticians, interceptions aren't logical truths, yet their truth is logically necessary.

Still finer refinements are found. Hilary Putnam calls all synomic interceptions logically necessary, since all are true in all logically possible worlds. Saul Kripke says a coreferential interception like BBI is metaphysically, not logically, necessary, because, unlike a copredicational interception (VPI), BBI isn't synonymous with its logical correlate, BBL. ${ }^{8}$ Actually, whether predicational or referential, the necessity of a synomic interception is never logical, explained by syntax, but always lexical, explained by

8 Hilary Putnam, Realism with a Human Face (Cambridge: Harvard University Press, 1990), pp. $57 \mathrm{ff}$.

(C) Blackwell Publishers Ltd. 1999 
semantic conventions governing the sentential material. ${ }^{9}$ Lumping these necessities together makes their being true in all possible worlds more significant than why they are. The contrast we ought to be marking is between the essential translinguistic structural constraints on all rational speech and the arbitrary constraints peculiar to a notational convention.

Another symptom of assuming interception synonymy is supposing (more or less) that, were it not for Gödel, we might sensibly regard all mathematics as really just a branch of logic, an outgrowth fed by a few deft definitions. The myth of interception synonymy trivializes the uniqueness of logical form. Gödel presented a proof about proof, about the epistemic limits of logic to justify our mathematical beliefs. The epistemic shortfall is an effect of shifting to extrasyntactic truth. The myth masks the import of that. It makes term synonymy a surrogate for logical form. It allows logical syntax no more import for sentence meaning than the merest notational coincidence.

This is no simpleminded mistake. Synomic interceptions flunk every test of sentence synonymy - except for the only measure that has mattered to logicians. Logical sentences are deemed synonymous with their synomic interceptions because their strict, literal readings state the very same fact: the same objects are denoted, the same properties are predicated of them, the same language-independent reality is asserted. Interception nonsynomy means that sentences having identical translinguistic factual content may differ in semantic context, cognitive, informational meaning. That possibility is prima facie implausible; upon further reflection is looks paradoxical, and then unintelligible., A whole lot of plausible theory, linguistic, logical and philosphical entwines with the assumption of interception synonymy. Extracting it means realigning lots of other ideas. We'll content ourselves attending only to some material aspects of logical form. ${ }^{10}$

9 Kripke thinks copredicational interceptions cannot be news for someone who understands the words well enough to understand the sentence, whereas coreferential interceptions can. The alleged epistemic contrast is unreal and irrelevant. 'Greece is Hellas' and 'Greeks and Hellenes' are equally newsworthy or not, whoever you are. The epistemic contrast catching Kripkean (and Fregean and Quinean) eyes is not semantic or syntactic; it's not between names and prdicates. It's that, generally, our cognitive relations with the properties and abstract objects we name and predicate are unlike our cognitive relations with the concrete individuals we name and do not predicate.

${ }_{10}$ Other matters are confronted in my 'Identity', 'The Synonymy Antinomy', 'Meaning as Explanation', The Journal of Value Inquiry, forthcoming, and 'Quotation Apposition', The Philosophical Quarterly, Oct. 1999. See also 'Distinguishing mathematics from Logic', Paideia Achive, http://www.bu.edu/wcp/, 1999. 
Interception nonsynonymy is explicable only by attributing semantic import to the distinctive feature of syntactically secured truth that interception eliminates: term recurrence. Some recognition of term recurrence's essential role in inferential relations is as ancient as the dictum that no term pivotal to the validity of an inference appears in the conclusion unless it appears in a premise. ${ }^{11}$ Why is term (and sentence) reoccurrence a (the?) key feature of logical truth and formal inference? Because logical constants construct statements incapable of falsehood only if pairs of their pivotal extensional relata (terms, sentences) are extensionally identical. This coextension is ensured without extrasyntactic assumptions only if the relata are tokens or replicas of the same expression. Any other semantic linkage is optional, an effect of something contingent, empirical, not intrinsically rational or knowable a priori, a source of uncertainties.

The semantic import of term recurrence in logical sentences is little noted, and, like interception nonsynonymy, often implicitly denied. That goes along with various other more familiar and less reputable notions. Among the more radical of the lot is the (barely intelligible) thesis that logical truth and necessity don't categorically differ from synomic truth (VPI, BBI) and lexical necessity, because at bottom they are all explained by notational conventions. This assumes that the semantics of the syncategorematic (logical constants, connectives, operators) is just like that of terms. Of course, only convention determines whether conjunction is symbolized by an ampersand, a dot, or 'and', or 'und', or sentence juxtaposition. Still, the semantic import of those symbols differs from that of terms in one whopping way. Synonymous logical constants are freely interchangeable in logical truths without affecting sentence syntax or sense; synonymous terms are not. Consider:

MHL: If Mary and Harry sell spigots, Harry sells spigots MH\&: If Mary \& Harry sell spigots, Harry sells spigots MHI: If Mary and Harry sell spigots, Harry sells faucets.

MHL and MH\& are fully synonymous logical truths of form, ' $(A m . A h) \rightarrow A h$ '. MHI is not a synonym; it is an extralogical truth of form ' $(A m . A h) \rightarrow L h$ '. This contrast is no matter of convention. Term recurrence is required for syntactically securing coextension

${ }^{11}$ Term recurrence is implicitly required and realized by the sentence recurrence required for inferences in sentential logic.

(C) Blackwell Publishers Ltd. 1999 
of the syntactic relata. The only uniformity demanded of the representation of the syntactic relations themselves is in the structure displayed in the sentence material: the same flankings by the same terms.

Conventionalists try to explain their proposal by talking about uninterpreted formal systems and games like chess. However, since none of these conventional structures express anything, let alone something true or false, they're hintless how logical truth is explained by conventions. So conventionalists turn to definitional truths to explain their conception of logic. To no avail. A synomic truth shows its conventionality by being inexpressible without using the terms whose conventions explain its truth. Meanwhile, logical truths exhibit their independence of conventions by being freely translatable. What explains their truth is their multirealizable structure, not any convention. Similarly, if 'Lead is a metal' is called true by definition, we're talking of a real definition, not a truth explainable by conventions. It is a multiexpressible, readily translated truth, independent of any specific terms, and inexplicable without reference to extrasentential reality.

Disregard for the import of term recurrence is entwined with another, more pervasive, notion, the conception of logical form as principally the form of a thought, and only incidentally, if at all, the form of a sentence, the expressional matter. At play is a 'picture' of a proposition, not some one well defined thesis.

A proposition is something expressed by words, but somehow independent of any word. It is a multiexpressible thought content, a transnotational truth value bearer, identifiable by and with many actual or possible sentences, yet distinguishable from any sentence. Sentence tokens are concrete, sense-perceptible particulars, and sentence-types are abstractions of such shapes. Syntactic form is preserved in interlinguistic translations having all sorts of lexical transformations and transpositions, So syntax isn't identifiable with the sequence of a sentence's lexical constituents or any perceptual, spatial or temporal configuration, not even an abstract one. Grammar isn't geometry.

If a truth (a true proposition) is the factual content of a sentence, so a logical sentence and its synomic interceptions all state the same truth, then the syntax that suffices to explain truth is the structure of the factual content shared by a logical truth and its interceptions. Then, at the level of logical form, term recurrence is really recurrence of term meanings: synonymous terms are the same term. 
Logical syntax is surely abstract. Still, it is an ordering of expressional matter, not of supra-sense-perceptible items, pure meanings. Syntactic categories are functional categories. They characterize the role an expression plays in the organization of expressional elements constituting a sentence. Though that role is not something sense-perceptible or spatio-temporal, what has the role must be a symbol, something individuated by its matter and not (just) by what it symbolizes.

Consider: while we seem to meet no end of difficulties in specifying the syntactic role of 'the', 'if', 'not', 'or', 'any', etc., the project makes progress. However, there's no beginning to specifying the syntactic role of their meanings. The idea of a meaning having a syntactic role is more than a little mystifying, especially when an expression has little meaning other than its syntactic role. Among the many mysteries would be very idea of definition, since individuation of terms by their meaning seems to imply that ' $\mathrm{G}={ }_{\mathrm{df}} \mathrm{H}$ ' entails “ " $\mathrm{G}$ " =" $\mathrm{H}$ ”, which makes it a marvel how we ever introduce a new term or explain the meaning of an existing one.

Put this another way. The picture of interception synonymy effectively conflates the notions of logical and lexical necessity, of truth secured by syntax and truth secured by semantics. The picture says that the synonymy of a synomic interception only explains why the interception's deep structure is logical; the interception's necessity is explained by its underlying logical syntax, not by the contingent synonymy.

Actually, with atomic synomic truths like VPI and BBI, if their necessity were not explained directly by the synonymy, their equivalence to their logical correlates would be inexplicable and indemonstrable. Logical and interceptional truths differ in meaning, because their meaning, what explains their factual content, is the explanation of their truth and necessity. We don't much understand a logical sentence until we (however tenuously) catch on that syntax is doing all the work. You don't really get what a synomic truth says without some sense that it's all a matter of word meanings. Both explanations are intrasentential, unlike that of synthetic sentences (physics, math, etc.) whose truth wants explaining by some extrasentential reality.

We may and perhaps must talk of a proposition's logical form as well as a sentence's, but the former notion feeds off the latter. Otherwise, truth due to syntax become indiscriminable not only from truth due to semantics, but also from truth about syntax. Sentences having logical form, like: 
ERL: Ed's reigning or Ed's not reigning

EKL: If Ed is a king and kings reign, Ed reigns

Form: $p v \sim p$

Form: $\operatorname{Ke} \&(\mathrm{x})(\mathrm{Kx} \rightarrow \mathrm{Rx}) \rightarrow \mathrm{Re}$

are not statements about logical form, like:

ERA: Ed's reigning or the contradictory is true Form: pvq EKA: The conjunction of Ed's being a king and kings' reigning implies that Ed reigns

Form: Ic (a predication of 'implies that Ed reigns' (I) on the singular term, 'the conjunction of . . . ' $)^{12}$

The L (logical) and A (about) correlatives surely aren't synonymous, yet their truth conditions, factual content and entailments seem identical. In that sense, despite disparate syntax, they state the same proposition. Similarly (albeit more tendentiously) with:

GGL: Germany is Germany

DDL: Deutschland ist Deutschland

GIA: Germany is identical with itself

GSA: Germany is self-identical

DIA: Deutschland ist mit sich selbst identisch Form: Igg.

The English sentences and their German version are semantic equivalents by having the same syntax and semantically equivalent words. So too for the synonymy of GIA and GSA. The factual equivalence of the $\mathrm{L}$ and $\mathrm{A}$ sentences is inexplicable that way. ${ }^{13}$

$\mathrm{L}$ and $\mathrm{A}$ sentences express the same proposition despite differing in both syntax and terms because the A sentences describe the formal relations their L mates display. An L sentence exemplifies a sentential structure necessitating the truth it states, whatever its terms say. The A sentences state truths about logic. Their necessity is not in their own syntax, but in the logical fact they state. They are true because their terms name or predicate the syntactic relations in their L correlates, a syntax that explains its own truth. Again:

MPL: $\mathrm{p} \&(\mathrm{p} \rightarrow \mathrm{q}) \rightarrow \mathrm{q}$

MPA: From a conditional and its antecedent, the consequent follows

12 The point here is unaffected by taking ' $\mathrm{I}$ ' to be the relational term 'implies', with 'e' a singular term for 'that Ed reigns', so the form would be: Ice. Use of Russellian or other parsings of the singular term(s) is also immaterial.

13 See below, and also 'Identity and syntax'. 
MPA states a principle of logic, not because of its own form, but because, through its terms it rightly says that sentences having the form of MPL are ipso facto true.

The sentential character of logical form may be admitted, but underrated by taking talk of sentential form to be metaphorical, which is bound to be the cost of disregarding the syntactic role of term recurrence and the replication of material elements it involves. Again, elsewhere the matter of a sentence seems to know no bounds. Even granting (as some may not) that sentences must be and consist of reproducible, perceptible elements, still the representation of syntactic relations may seem unlimited, just as any symbol might be a term for any object or property. It may seem that securing coextension by material replication is no less a matter of convention than any other representational device. It may seem incredible that a truth could constrain its representation.

This train of thought may start from the ever present possibility of polysemy. The sentence:

SBL: If she threw a ball, she threw a ball

is extralogical if 'ball' is meant two ways, logical if but one. So, the thought goes, what determines logical form is recurrence of how the words are meant, not the mere reoccurrence of a symbol. The two readings are distinguishable only semantically, not syntactically. The synonymy of the first and second 'ball' is determined extrasyntactically, just as their nonsynonymy is, and just like the synonymy of 'VP' and 'vice-president'. As a matter of logic, any symbol could have or lack any of many meanings. Logicians may by fiat finesse the interpretational uncertainties attending ambiguity by stipulating that all symbols in their systems are strictly univocal. That stipulation is just another extrasyntactic convention. And so is any rule for reading repetitions of a symbol the same. Some ciphers lack any such rule. The reading of a symbol may instead be determined by its position in the sequence of its appearances in a message. Self-identities might be written as ' $\mathrm{b}=\mathrm{c}$ ', and modus ponens represented as ' $\mathrm{p} \&(\mathrm{q} \rightarrow \mathrm{r}) \rightarrow \mathrm{s}$ '. ${ }^{14}$ As things are, as we are, with our various communicational goals, with all the psychological and technological constraints on reproducing and perceiving symbols, storing and

14 See John Searle, 'Proper Names', Mind LXVII, 266 (1958), pp. 166-173.

(C) Blackwell Publishers Ltd. 1999 
processing information, coordinating behavior, and so on, it's obviously sensible to let the reappearances of a shape or sound have the same sense, except when we aspire to disguise our meaning. All this is rational, and natural, and normal, but it is all conditional on the empirical contingencies of our interests and capacities. No inherent necessity inhabits any of this. Logical truth cannot be constrained by matter.

This line of thought is tangled and knotted. First off, polysemy provides no argument for the conventionality of the principle that tokens (or replica) of a term are synonymous, for polysemy presupposes just the opposite. Words and sentences wouldn't be ambiguous if the tokens didn't carry the senses of their type. Unless an utterance has multiple meanings just by its grammar (syntax and vocabulary), apart from its speech context and speaker's intention, a speaker could not choose to intend one meaning rather than another and the audience couldn't wonder which one got chosen..$^{15}$

The possibility of polysemy presents epistemological problems, not formal, logical ones. Here logic is like math or mechanics. Arithmetic does not determine what is meant by any particular token of ' $55 \times 11=605$ ', but only that, if what is meant is that $55 \times 11=605$, the statement is true. Likewise, logic doesn't determine whether an utterance of 'If she threw a ball, she threw a ball' has form ' $p \rightarrow p$ ' or ' $p \rightarrow q$ '. The point of principle is only that, when SBL is used to say that if she threw an event, she threw an event, its form is ' $p \rightarrow p$ '; when SBL is used to say that if she threw an event, she threw an object, SBL is extralogical, form ' $p \rightarrow q$ '. How we are to discover which was meant is no concern of logic. Logicians and mathematicians are rightly concerned to free themselves of the irrelevant epistemic complications brought by ambiguity, so they exclude lexical and syntactic ambiguity from their formal systems. Their rule restricting each term type and its tokens to one meaning is a convention that neatly eliminates irrelevant epistemological questions. That convention presupposes the principle that every token of a term has the standard meanings of its type. The convention cannot imply that the principle is itself a convention.

Next, note that positional ciphers are not examples of nota-

15 Tokens may have meanings lacked by the type and other tokens, meanings peculiar to a speech context or speaker's intent. Generally, even these senses are available only because the token has the standard meanings of its type. 
tions without synonymous symbol tokens. They illustrate only that (e.g.) word sameness may be sameness of shape-in-nth-position, or something other than same printer's characters. Representing self-identity with 'Dogs are cats' is not in principle unlike representing it with 'Dogs are dogs', or other more visually distinctive shapes. Term types and token sameness cannot but be specifiable in very various ways, including ways alien for humans. Doubtless, criteria for individuating word types may have ineliminable conventional aspects, but that doesn't argue for the conventionality of the principle that words and sentences are rule governed repeatable symbols whose replicas have the same meaning. ${ }^{16}$

The constraints on representation are not all merely pragmatic or technological. Notice: positional ciphers demand that all discourse be jacketed to discrete messages to determine sequence and position. Worse, such ciphers permit nothing comparable to enquoting a token to refer to its type or another token. That makes the cipher unlearnable, undiscussable, unworkable on its own, without a background natural language. More generally, a notational system must have symbols that can be used selfrepresentationally if elemental metalinguistic speech, like ostensive definitions, is to be possible.

Misunderstanding of this last matter is another cause and consequence of missing the significance of term recurrence. Many respected theorists assume that QMi:

QMi: ' $\mathrm{E}_{\mathrm{x}}$ ' means (says, refers to) $\mathrm{E}_{\mathrm{x}}$

represents only a convention, not a (meta) logical principle. Allegedly, QMi sentences are syntactically indistinguishable from the more general form:

QM: $\mathrm{E}_{\mathrm{x}}$ means $\mathrm{E}_{\mathrm{y}}$.

The idea is that semantic claims - all QM sentences including QMi sentences - are always contingent, true by convention, never logical, true by syntax. This isn't the triviality that quotation marks, underlining, italicizing, etc. are alternative conventions like the ampersand, dot and 'and'. Rather, the claim is that a linguistic expression is not inherently a representation of itself as well as whatever else it symbolizes. Brown.

16 This matter needs no belaboring for grue groupies and browsers of books Blue and 
As with other cases of term recurrence, confusion about QMi comes from forgetting that syntactic relations are intrasentential, and focusing instead on the possibility of using the same symbol in separate senses. Certainly, an ostensible QMi sentence is contingent, just an instance of $\mathrm{QM}$, if the referent subject is some token or type in some other discourse. QMi is a (meta) logical truth only if corepresentation is secured syntactically by indexing quotational reference self-referentially, intrasententially, to designate the expression (type) replicated in the predicate.

Denial of QMi's logical truth underpins suppositions such as that the tripartite equivalence:

Ed's reigning $\equiv$ it is true that Ed's reigning $\equiv$ 'Ed's reigning' is true

presupposes some empirical contingency like: 'The English sentence, "Ed's reigning" means Ed's reigning'. Yet, if that were so, that premise wouldn't suffice, for there would then be need to premise:

The English sentence ' "Ed's reigning” means Ed's reigning' means the English sentence 'Ed's reigning' means Ed's reigning.

And so on. QMi cannot be needed as a premise in inferences according with it, any more than modus ponens need be a premise in inferences conforming to that principle. Instead, the validity of the inference is the syntactically secured truth of the conditional representing it.

The preceding prepares us to appreciate that, while mathematics might properly be called a formal discipline, it cannot be in the sense that logic is. Consider:
A: $7=7 \quad 7$ is 7
B: $7=\mathrm{VII} \quad 7$ is VII
C: $15-7=15-\mathrm{VII}$
D: $15-7=2^{3}$
E: $15-\mathrm{VII}=2^{3}$
F: $15-7=12-2^{2}$.

Both sentences of A may be taken as logical truths, and no more mathematical than 'A rose is a rose' ranks as a botanical truth, for their objectual references are irrelevant to their truth. Synonym subbing in A yields B, sentences most naturally said and read as synomic truths, rather than mathematical or logical ones. 
Similarly, C looks like only a more complex version of B, a synomic sentence useable for explaining the notational equivalence of the numerals ' 7 ' and 'VII'.

What intuitively seems distinctively mathematical, not (or not merely) logical or notational, is D. And, unlike a logical truth, D seems synonymous with its substitution resultant, E. They differ only notationally. F, I assume, is not synonymous with $\mathrm{D}$ or $\mathrm{E}$; it states a different mathematical fact. But D, E, and F carry the same mathematical necessity, a necessity differing in kind from that of A, and that of B and C. So, unlike logical truths, synonym subbing in extralogical mathematical statements transmits syntax, sense, and mathematical necessity.

Mathematical truth is not syntactic. Term recurrences play no essential role in mathematics. Uniform term substitutions in mathematical sentences can transform truth values, while nonuniform synonym subbings have no mathematical import. Construction of mathematical synonyms may be constrained by computational considerations: substituting 'VII' for ' 7 ' makes arithmetic operations typographically tougher. But the sense of the mathematical sentences isn't touched. ${ }^{17}$

Mathematical necessities are not logical necessities, but neither are they lexical necessities. Stipulative and nominal definitions have no more utility in mathematics than in other disciplines. They determine the notational tools but not any substantive truths. Such definitions license synonym substitutions wherein one name is replaced by another name, with no mathematical progress made thereby. Such definitions can explain only synomic truths, like ' $7=$ VII' and ' $15-7=15-$ VII', not contentful, transnotational mathematical truths, like ' $15-7=2$ '.

Nor are conceptual definitions or analyses of much help, if a concept is anything psychological like a belief (and if it's not, I don't know what it is). ${ }^{18}$ Whatever the concept, the question is open whether our concept is correct. That competent mathematicians or people generally accept the concept may be reason

17 Arguably, ' $156 / 13=12$ ' is not fully synonymous with 'CLVI/13=12'. In both, the quotient (number) named by the right hand term is the resultant of the operation represented by the left side of the equation, but only in ' $156 / 13=12$ ' is the quotient term (numeral) the resultant of an operation with the dividend and divisor terms (numerals). Arguably, the computational intranotational relations of the Arabic system are implicit in the informational content of the Arabic equation.

${ }_{18}$ Here my curt dismissal of concepts can express only my preferences for the most common, ordinary use of 'concept of X', where it means some set of core beliefs about $\mathrm{X}$.

(C) Blackwell Publishers Ltd. 1999 
to think it likely correct, but such evidence is not dispositive. In any case, the subject matter of mathematics is not people's beliefs about the matter.

The definitions needed for mathematical derivations are real definitions of (e.g.) number, not nominal definitions of the word 'number' that provide a synonym (another name for the thing), nor conceptual definitions specifying our core beliefs about number. As in any science, progress may come from real, analytical definitions wherein an object (property, relation, etc.) directly referred to by a name is analyzed and described by predication of its core properties. Such definitions are only secondarily truths about language. Their correctness and utility isn't explained intrasententially.

The property named 'circularity' attributed with the predicate, 'circle', (or 'circular') is identifiable in dozens of ways, by referring to any of the various properties possessed by all and only circles. None of the names for those properties is a synonym of "circularity'. Some of specifications of the property (like reference to equidistant center) are, for historical and psychological reasons (like perceptual salience), more naturally and properly called 'definitions' of the natural language term. Other specifications (like reference to pi) seem less like definitions and more like discovered essential properties. What is treated as definitional in a formal theory may vary with the specific aims of the theory.

There seems little profit in speculating which predications are (more) essential to our concept of circularity, and which are readily revised or rejected in response to the next revolution comparable to the coming of non-Eucledian geometry. We certainly can't know in advance (it may take genius to vaguely imagine) the next great fundamental discovery that could justify wondering (1) whether denying some hitherto accepted property of circles is a change of language, of meaning or reference, or a change of belief about the same old referent, and (2) either way, whether we should accept that denial or the denial of some other entrenched predication that raises the first question. We don't daily have cause for such doubts, but, unlike doubts about the existence of this or that ontological kind (physical objects, other minds, etc.), we do well to recognize that, generally if not universally, ultimately we cannot know in advance, by introspection or otherwise, what we are referring to. Unlike radical scepticism of the reality of an ontological kind, the evidence piles up at a faster rate every decade that we are sometimes shockingly wrong 
about the identity of the property we refer to with some common name. We may later learn that some of that evidence is less conclusive than we had thought, but that discovery may be further evidence of a greater real fallibility. ${ }^{19}$

These epistemological aspects are secondary. Sentence meaning explains method of verification, not vice-versa, and mathematical sentence meaning isn't structured like that of logical and synomic sentences. Mathematical truth isn't provable by logic and synonym subbing, because it's not explainable intrasententially. Like logical truths, mathematical truths transcend the notations with which we express them. Unlike logical truths, their necessity isn't explained by material features of their representation, nor are they truths about such necessities and the structure of propositional thought.

The great Fregean project is misguided. From the outset it elides or denies the formal divide between syntactic and synthetic truth. This is explicit in its interpretation of the mathematician's equality sign, '=' as synonymous with (indeed the very same sign as) the logician's identity sign, ' $=$ '. That's like mistaking the mathematician's addition sign, '+', for the logician's conjunction sign, ' $\&$ '.

Logical constants represent syntactic operations or relations between syntactic objects: sentences, names, predicates, and the like. The operations and relations of mathematics are objectual, not syntactic. Its ' + ' and ' $=$ ' represent operations or relations between mathematical objects, numbers, quantities, and the like, not between members of linguistic categories like sentences and terms.

We may speak of adding (dividing, subtracting) sentences in various senses. When we intend some kind of computing, the relata of ' + ' are not sentences, sentinels or such things, but numbers, like the numbers of some sets of sentences. So too, the arithmetic sign of equality rarely appears flanked by two names, except in notational truths like ' $7=\mathrm{VII}$ ', or conclusions of proofs of some substantive mathematical proposition by demonstrating its truth functional equivalence with a triviality like ' $7=7$ '. ${ }^{20}$ In ' $19-12=7$ ', ' $19-12$ ' is not a name of anything, no

19 See my 'Socratic Scepticism', Metaphilosophy 24 (1993), 344-62.

20 It is a relevant datum that it sounds somewhat odd, strained (I don't say wrong, absurd or nonsensical) to use the word 'equals' in genuine self-identities and alter-identities, flanked by genuine names.

(C) Blackwell Publishers Ltd. 1999 
more than nineteen minus twelve'. These are notationally equivalent predicative expressions, definite descriptions uniquely specifying an arithmetic object. These expressions describe a relation between numbers, an 'operation' with/on numbers: the subtraction of 12 from 19. The 'result' of that 'operation' is a quantity equal in its number to the number also referenced directly by the names ' 7 ', 'VII', 'seven'. Those terms canfunction predicatively, as in, 'That sentence has seven words'. In the statement, 'The subtraction of 12 from 19 equals (is equal to) 7', the property, equality with 7 (being equal in number to 7), is predicated of the relational object, the subtraction of 12 from 19. That statement is equivalent to: 'Seven is equal to 19-12', wherein the property, being equal to the subtraction of 12 from 19, is predicated of the object named 'seven'.

The logician's '=' marks a relation between two names or other direct, rigid, codesignators, not between their objectual referent(s). In sentence BW ('Batman is Bruce Wayne') a twice named object is objectually referenced, but no objectual relation or relational property is predicated of it. There is no predicate in BW. Just two names and a copula, a syntactical marker. There is a predicate in BIW ('Batman is identical with Bruce Wayne'), but the relational predicate, '(is) identical with ___,', is comparable to the relational predicate, '(is) conjoined with

', which attributes a syntactic relation (called 'conjunction') between two sentences instead of two names. We may also properly speak objectually of conjoining two facts, but nothing extralinguistic is being described. So too, BW and BIW are factually, objectually identical, the same as BB ('Batman is Batman'). The distinction in semantic, assertorial content is intrasentential: it's not anything in the world the sentence's terms objectually reference. $\mathrm{BB}$ and $\mathrm{BW}$ contain direct coreference. BB secures it syntactically. BW's coreference is a consequence of extrasyntactic factors. BIW codesignates by direct reference and by predication of a linguistic relation. In each case the sentence's content is explained by features of the sentential matter which necessitate its truth. They talk about an object, but no language independent fact about it is stated. Absent the linguistic matter, there is no content to the identity.

Mathematical truths are predications of extralogical and extra-linguistic properties. They might be truths about some 
extrasyntactic structure of quantity or space. They are not truths because of or about the structure of talk or thought. Whatever else they may be, their surviving synonym substitution means they are not formal truths like those of logic. ${ }^{21}$

${ }^{21}$ This work owes much to the efforts of Bredo Johnsen and David Massie. 